www.jmscr.igmpublication.org Impact Factor 5.244

Index Copernicus Value: 83.27 ISSN (e)-2347-176x ISSN (p) 2455-0450 crossref DOI: _https://dx.doi.org/10.18535/jmscr/v4i12.28

Journal Of Medical Science And Clinical Research

\title{
Sputum Smear Conversion Time of HIV Infected and Uninfected Patients with Rifampicin and Isoniazid Mycobacterium tuberculosis gene Mutations in Western Kenya
}

\author{
Authors \\ Shiluli, Clement ${ }^{1,2}$, Ouma, Collins ${ }^{2,3}$, Vulule, John ${ }^{1}$, Khayumbi, Jeremiah ${ }^{1}$, \\ Murithi, Wilfred $^{1}$, Musau, Susan ${ }^{1}$, Okumu, Albert ${ }^{1}$ \\ ${ }^{1}$ Kenya Medical Research Institute (KEMRI)/Center for Global Health Research (CGHR), Kisumu, Kenya, \\ ${ }^{2}$ School of Public Health and Community Development, Department of Biomedical Sciences and \\ Technology, Maseno University, Maseno, Kenya \\ *Correspondence School of Public Health and Community Development, Department of Biomedical \\ Sciences and Technology, Maseno University, P.O. Private Bag, Maseno \\ Email: clementshiluli@yahoo.com,Mobile Number+254 723474168
}

\begin{abstract}
Background: In 2013, an estimated 9.0 million people developed tuberculosis (TB) and 1.5 million died from the disease, 360000 of whom were HIV-positive. A major challenge to TB management is the multi-drug resistant (MDR) TB strains and HIV. There are few studies in western Kenya on the specific mutations underlying resistance to rifampicin (RIF) and isoniazid (INH) and the time to sputum smear conversion, especially in HIV-infected patients.
\end{abstract}

Methods: We therefore studied sputum smear conversion time in TB and HIV co-infected patients with previously confirmed rpoB, katG and inhA Mycobacterium tuberculosis gene mutations. Drug sensitivity tests and line probe assays had been performed previously on sputum samples from participating patients. Samples with discordant results were further sequenced to confirm rpoB, $\mathrm{kat} G$, and inhA gene mutations that have been associated with RIF and INH mutations. Gene mutations were classified into three categories based on specific codons with mutations on the rpo B, kat $G$, and in hA genes as follows, MDR-TB, RIF mono-resistant (RMR) $T B$ and isoniazid mono-resistant (INHMR) TB. Ziehl-Neelsen (ZN) microscopy was done on sputum samples from enrolled patients on two occasions; during first and follow-up visits at respective health facilities. The period of follow-up was less than one year. Smear results were available for 16 patients with confirmed drug resistant TB. The smear conversion rate was determined by dividing the number of patients who had a negative smear during follow-up and the number of smear positive patients on first visit and multiplying by 100. Spearman's correlation coefficient was used to assess the strength of associations between continuous and ordinal variables.

Results: The smear conversion rate for participating patients was as follows; RMR-TB =100\%, INHMR-TB= $60 \%$ and $M D R-T B=67 \%$. All the patients had positive Mycobacterium tuberculosis cultures. There was positive correlation between follow-up days and $Z N$ smear results, $\left(r_{s}(14)=0.097, p=0.721\right)$.

Conclusion: Sputum smear conversion time can be used in monitoring drug resistant TB in both HIV-infected and-uninfected patients.

Keywords: Tuberculosis, HIV, MDR-TB, isoniazid mono resistant, rifampicin mono resistant gene sequencing. 


\section{Introduction}

In 2013, an estimated 9.0 million people developed tuberculosis (TB) and 1.5 million died from the disease, 360000 of whom were HIVpositive ${ }^{(1)}$. Globally, approximately 480000 cases of multi-drug resistant TB, defined as TB caused by strains of Mycobacterium tuberculosis (M. tuberculosis) resistant to at least isoniazid and rifampicin, were reported $^{(1)}$. In Kenya, the prevalence of $\mathrm{HIV}$ is $5.6 \%$ and HIV co-infection rate is $35 \%{ }^{(2)}$. A major challenge to $\mathrm{TB}$ management is the presence of MDR-TB(because patients are treated with expensive second-line drugs) and HIV, which is a known risk factor for TB ${ }^{(3,4)}$. Rifampicin and isoniazid are important drugs in first-line anti-TB treatment irrespective of HIV status ${ }^{(3)}$. Drug resistance to at least one or both has been associated with poor response to treatment ${ }^{(4)}$. It is recommended that MDR-TB should be monitored routinely through drug susceptibility tests (DST) ${ }^{(5)}$. However, recent studies have reported more than $90 \%$ agreement between DST and smear results in MDR-TB patients being treated for up to a period of 27 months ${ }^{(4,6)}$. Sputum smear microscopy to monitor treatment response measure is therefore practical in resource limited countries and provides rapid results, is inexpensive, easy to perform, does not require complex laboratory equipment (7). Rifampicin resistance occurs as a result of mutations on the $r p o B$ gene that encodes the $\beta$ subunit of the RNA polymerase ${ }^{(8) .}$ Resistance to isoniazid is classified into either high or low level resistance depending on the type of gene mutations ${ }^{(8)}$. Isoniazid is activated by the enzyme catalase peroxidase, encoded by katG and mutations on this gene lead to high-level isoniazid resistance ${ }^{(8)}$. The inhA gene encodes an enoyl acyl carrier protein reductase involved in fatty acid synthesis and isoniazid interferes with this process ${ }^{(8)}$. Mutations on this gene lead to lowlevel isoniazid resistance ${ }^{(9)}$.There are few studies on the specific mutations underlying resistance to rifampicin and isoniazid and the time to sputum and liquid culture conversion, especially in HIV- infected patients ${ }^{(10)}$. We therefore studied patients with previously confirmed rpoB, kat $G$ and inhA M. Tuberculosis gene mutations and sputum smear and liquid culture conversion time in TB and HIV co-infected patients in western Kenya.

\section{Materials and methods \\ Study site}

This study was conducted between 2012 and 2014. This is a reference laboratory for drug resistant $M$. tuberculosis for health facilities in more than five counties in d western Kenya. According to the Ministry of Public Health and Sanitation guidelines, the following regimen, administered for 9 months is recommended; for INH resistance with or without streptomycin (STR) resistance: rifampicin (RIF), pyrazinamide (PZA), ethambutol (EMB) and levofloxacin (LFX); for INH and PZA resistance: RIF, EMB and LFX; for INH and EMB resistance: RIF, PZA and $\mathrm{LFX}^{(11)}$. The treatment regimen of rifampicin mono-resistant (RMR) TB and MDR-TB consists of the following; for 6 months; kanamycin (KM), prothionamide (PTO), LFX, cycloserine (CS), and EMB or PZA followed by PTO, LFX, CS and EMB or PZA for 18 months ${ }^{(11)}$.

\section{Study Design}

The study quantified the association between MDR-TB, rifampicin and isoniazid resistanceconferring mutations and treatment outcome. Specific mutations on the $r p o B, k a t G$, and inhA gene mutations associated with rifampicin and isoniazid resistance were classified into three categories as follows, MDR-TB; sputum samples with mutations on rifampicin and isoniazid resistance conferring genes, rifampicin monoresistant $\mathrm{TB}$; sputum samples with rifampicin resistance conferring genes and isoniazid monoresistant TB; sputum samples with isoniazid resistance conferring genes. 


\section{Laboratory methods}

DST, LPA and Gene Sequencing

Drug sensitivity tests using the BACTEC ${ }^{\mathrm{TM}}$ MGIT $^{\mathrm{TM}} 960$ SIRE kit (BD Diagnostic systems, Baltimore, Maryland, USA) and line probe assays using the MTBDR plus v2.0 kit (Hain Life science, Nehren, Germany) had been performed on sputum samples from participating patients. Discordant samples were further sequenced using the Big Dye ${ }^{\circledR}$ Terminator v3.1 Cycle Sequencing Kit to confirm rpoB, katG, and inhA mutations. During follow-up visits to the health facilities, approximately $5 \mathrm{ml}$ of sputum sample was collected from each patient.

\section{ZN Microscopy}

Samples were transported to the referral laboratory for Ziehl-Neelsen (ZN) microscopy which was done by staining heat-fixed smears on microscopic slides for 5 minutes with carbolfuchsin (Sigma-Aldrich Co., St. Louis, Missouri, USA), decolorizing for 3 minutes, followed by counterstaining with malachite green (Sigma-Aldrich Co., St. Louis, Missouri, USA) for 1 minute. Sputum smear microscopy results were interpreted according to the International Union Against Tuberculosis and Lung Diseases (IUATLD) grading system ${ }^{(12)}$. The quality of results was ensured by having two independent microscopists read the slides. Results were entered into an Excel spreadsheet.

\section{Statistical Analysis}

The proportion of smear converted-patients was calculated by dividing the number of patients who had a negative smear during follow-up and the number of smear positive patients with confirmed drug-resistant TB and multiplying by 100 . Spearman's correlation coefficient was used to assess the strength of associations between continuous and ordinal variables.

\section{Results}

Smear microscopy conversion rates of TB patients with rifampicin and isoniazid conferring mutations

Ziehl-Neelsen (ZN) microscopy was done on two occasions; at initial visit for drug-resistant TB evaluation and during follow-up visits for monitoring disease progression at respective health facilities. Follow-up smear results for 16 patients were available as indicated in Table 1. Rifampicin and isoniazid conferring gene mutations were classified into three categories; rifampicin mono-resistant (RMR), isoniazid mono-resistant (INHMR) and multi-drug resistant gene mutations (Table 1). Sputum conversion rate was calculated as previously explained. There was positive correlation between the number of days between laboratory visits and $\mathrm{ZN}$ smear results, $\left(r_{s}(14)=0.097, p=0.721\right)$.

\section{Smear conversion time for HIV positive and} HIV negative patients with drug resistant TB

A total of 6 and 5 HIV positive and HIV negative patients respectively had smear converted as shown in Fig 1. The median smear conversion time was higher 6.5 months in HIV positive patients and 3 months in HIV negative patients (Fig 1). 
A

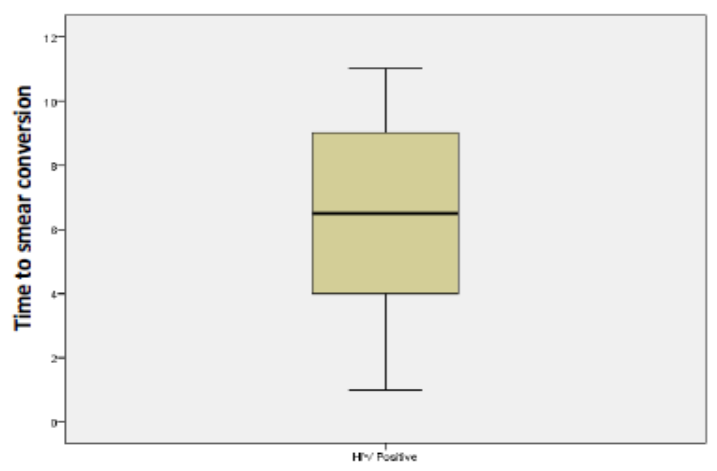

B

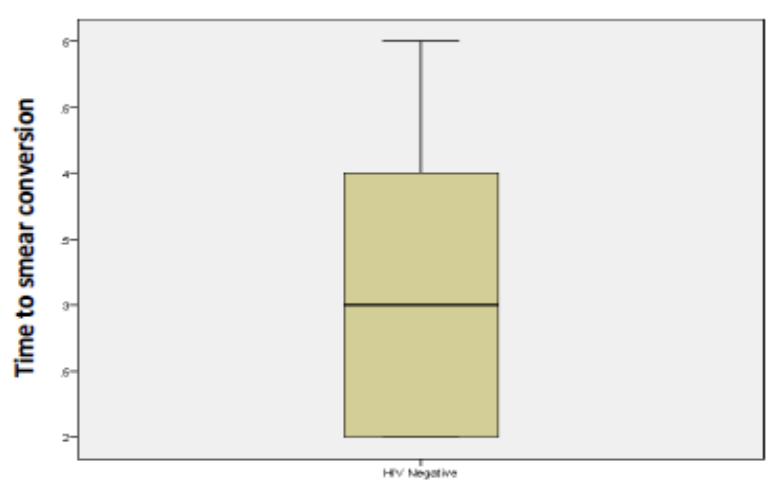

Fig 1: Sputum smear conversion time for HIV positive (A) and HIV negative (B) patients with drug resistant TB.

Table 1. Sputum smear conversion rates for HIV-infected (in bold) and uninfected patients with rifampicin and isoniazid conferring gene mutations at initial and follow-up visits to the health facility

\begin{tabular}{|c|c|c|c|}
\hline Amino acid modifications & $\begin{array}{l}\text { Initial } \\
\text { smear }\end{array}$ & Follow-up (Months) & Sputum conversion $(\%)$ \\
\hline H526Y & $1+$ & NEG, (11) & \multirow{2}{*}{100} \\
\hline S531L & $3+$ & NEG, (8) & \\
\hline
\end{tabular}

\begin{tabular}{l|lll} 
S315N & $3+$ & $3+,(8)$ & \\
S315T1 & $3+$ & $1+,(8)$ & 60 \\
S315T1 & $3+$ & NEG, (4) & \\
S315T1 & $1+$ & NEG, (5) \\
$\mathrm{C}^{2}-15 \mathrm{~T}^{a}$ & $1+$ & $\mathrm{NEG},(2)$
\end{tabular}

\begin{tabular}{|c|c|c|}
\hline S531L and S315T1 & $3+$ & NEG, (5) \\
\hline D516F and S315T1 & $3+$ & NEG, (2) \\
\hline S531L and S315T1 & $1+$ & NEG, (9) \\
\hline S531L and C-15T $\mathrm{T}^{a}$ & $1+$ & NEG, (1) \\
\hline S531L and S315T1 & $2+$ & $1+,(2)$ \\
\hline S531L and S315T1 & $1+$ & NEG, (3) \\
\hline Missing wt and S315T1 & $1+$ & NEG, (4) \\
\hline D516V and S315T1 & $2+$ & $1+,(2)$ \\
\hline Missing wt and S315T1 & $3+$ & $2+,(2)$ \\
\hline
\end{tabular}

NEG; Negative, wt; wild type, MDR; Multi drug-resistant, RMR; rifampicin mono resistant; INHMR; Isoniazid mono resistant. HIV co-infected codon mutations are shown in bold.

Amino acid abbreviations: S, Ser; T, Thr; R, Arg; L, Leu; V, Val; H, His; D, Asp; Y, Tyr; F, Phe.

${ }^{a}$ Cytosine $(\mathrm{C})$ to Thymidine $(\mathrm{T})$ position -15 nucleotide substitution for the inhAgene regulatory regio 


\section{Discussion}

Sputum smear microscopy is the primary method for monitoring treatment response in resourceconstrained countries ${ }^{(13)}$. In this study, we investigated the sputum smear conversion time in TB and HIV co-infected patients with confirmed mutations on rifampicin (RIF) and isoniazid (INH) conferring genes. Calculation of sputum conversion time is an important measure for determining the progress of treatment ${ }^{(14)}$.

Previous studies have shown that the H526Y and the S531L mutations are associated with higher minimum inhibitory concentrations for RIF and therefore poor drug response ${ }^{(3)}$. In our study, these mutations were identified in two HIV coinfected patients who had a $100 \%$ smear conversion rate at 11 months and 8 months respectively (Table 1). This finding emphasizes the importance of prolonged RIF TB therapy for improved treatment outcome ${ }^{(15,16)}$. However previous studies have shown that smear microscopy is less sensitive in HIV positive patients with MDR-TB and cure should be confirmed after obtaining at least 5 negative sputum culture results during 12 months of treatment ${ }^{(6)}$.

Patients with INH mono resistance have unfavorable treatment outcome and relapse ${ }^{(9)}$. Therefore it is expected that smear conversion time will be prolonged. In the present study, the sputum conversion rate for patients with INH mono resistant was $60 \%$ (Table 1). Our data indicated that 4 out of 5 HIV co-infected patients had the S315T1 mutation that has been associated with high level INH resistance, however, most patients with this mutation had smear converted at the time of follow-up. Patients with the S315N mutation had a $3+$ bacterial load even after 8 months of treatment. Therefore, it is anticipated that treatment of INH mono-resistant TB should be prolonged for an improved treatment outcome. This finding conflicts with previous studies that recommended replacing the 8 months WHO treatment regimen to a shorter treatment period of 6 months in new TB patients ${ }^{(8,9)}$. Our data showed that patients with this rare mutation responded poorly to treatment. Previous studies have showed that S315T1 mutations have been strongly associated with streptomycin resistance $(8,9)$. The smear conversion time reported in our study could therefore be influenced by Streptomycin and INH resistance occurring simultaneously. The $\mathrm{C}-15 \mathrm{~T}$ inhA promoter mutation was only present in one patient and the 2 month smear conversion rate was expected since this mutation has been strongly associated with low level INH resistance ${ }^{(9)}$. This finding concurs with previous studies in areas with increases cases of INH mono-resistance that found that the inhA mutation was rare as compared to the S315T1 mutation ${ }^{(9)}$.

Patients with the S531L and S315T1 and S531L and C-15T mutations had smear converted at 9 and 1 months respectively. Our study showed that only three MDR-TB patients had a bacterial load of between 2+ and 1+ at follow-up (Table 1) and this observation is expected because patients had only been treated for two months at the time of follow-up. In the present study the sputum smear conversion rate for patients with MDR-TB was 67\% (Table 1). A previous culture based study using smear microscopy to monitor the treatment outcome of MDR-TB in patients also found cure rates of $66 \%$ in retreatment cases ${ }^{(10)}$. However, studies in countries with high cases of drugresistant TB have reported that MDR-TB patients on treatment with first-line therapy had a high rate of TB recurrence and died within 4 years ${ }^{(6)}$.

Overall, we found that HIV positive patients required longer periods of time to smear convert. A previous culture study in the USA demonstrated a 9 months conversion time, however, since genotyping of sputum samples was not performed, it was known if this particular finding was as a result of acquired resistance, re-infection, mixed infections or laboratory cross-contamination ${ }^{(6)}$. In addition, the study involved TB patients not exposed to HIV infection ${ }^{(6)}$. Even though we found a short smear conversion time in HIV negative patients, studies have shown that 
microscopy particularly in HIV positive patients failed to precisely detect bacilli in culture positive sputum samples suggesting that smear monitoring alone of drug resistant TB should be used with great caution ${ }^{(10)}$. HIV infection has been associated with poor drug efficacy and this could be the likely reason for the long smear conversion time, however, a previous study documented that HIV positive patients with MDR-TB who were on early Highly Active Antiretroviral Therapy (HAART) had improved treatment outcomes as compared to a control group from the pre-HAART era ${ }^{(10)}$.

In the present study, we found positive correlation between the number of days of laboratory visits and reduction in bacilli load as determined by $\mathrm{ZN}$ microscopy. Studies have recommended that sputum culture conversion at 2 months can be used for monitoring treatment in patients with pulmonary TB.

Our data had several limitations, we were not able to determine the exact time at which the study patients had started treatment for drug-resistant TB and we had few study participants due to the low prevalence of drug-resistant TB in the study population, in addition, we did not have culture results data on follow-up patients. Our findings need to be supported with other similar studies in HIV endemic regions.

\section{Conclusion}

Sputum smear conversion time can be used to monitor drug resistant TB in both HIV-infected and -uninfected patients.

\section{Recommendation}

We recommend that sputum smear conversion time can be used to monitor drug-resistant TB in both HIV-infected and uninfected patients in poor resource countries, however, this should be done in comparison to reference laboratories that perform culture of Mycobacterium tuberculosis.

\section{Acknowledgement}

We thank the KEMRI/CGHR Center Director, Dr. Stephen Munga Dr Kevin Cain, Dr. Jamie Posey, Dr. Mellisa Wilby, Dr. Videlis Nduba, Dr. Steve Wandiga, and the KEMRI/CDC Tuberculosis research laboratory staff.

\section{Declaration}

\section{List of abbreviations}

CDC - Centers for Disease Control and Prevention

DNA - Deoxyribonucleic Acid

DLTLD - Division of Leprosy Tuberculosis and Lung Diseases

DST - Drug Susceptibility test

ERC - Ethical Review Committee

HAART - Highly Active Antiretroviral Therapy

HIV - Human Immunodeficiency Virus

INH - Isoniazid

INHMR - Isoniazid mono resistant

KEMRI - Kenya Medical Research Institute

LPA - Line Probe Assay

MDR - Multi drug-resistant

RMR - Rifampicin mono resistant

RIF - Rifampicin

TB - Tuberculosis

USA - United States of America

\section{Ethical Considerations}

Ethical approval was obtained from the Ethical Review Committee (ERC) of the Kenya Medical Research Institute (KEMRI) Nairobi, protocol title; Gene mutations associated with Mycobacterium tuberculosis resistance to Isoniazid and Rifampicin in a HIV-1 endemic population in western Kenya, Scientific Steering Committee (SSC) number 2854.

\section{Consent to Publish}

All authors have read the final version of this manuscript and have approved it for publication. Informed consent was obtained from patients on sharing findings from this research through publications. 


\section{Competing interests}

The authors declare that they have no competing interest.

\section{Author's Contribution}

The authors declare no conflict of interest. CS designed the study, collected and analyzed data and wrote the manuscript, CO and JMV revised the manuscript, JK and WM collected data and revised the manuscript, SM and AO revised the manuscript. All authors proof read the final version of the manuscript before submission.

Funding: This research was funded by a grant from the Association of African Universities (AAU) and the KEMRI/CDC Tuberculosis Research Laboratory

\section{References}

1. WHO. Global Tuberculosis Report 2014. Geneva, Switzerland: World Health Organization.; 2014.

2. KAIS. Kenya AIDS Indicator Survey Annual report. 2013.

3. Jamieson FB, Guthrie JL, Neemuchwala A, Lastovetska O, Melano RG, Mehaffy C. Profiling of rpoB mutations and MICs for rifampin and rifabutin in Mycobacterium tuberculosis. J ClinMicrobiol. 2014 Jun;52(6):2157-62.

4. Quy HT, Cobelens FG, Lan NT, Buu TN, Lambregts CS, Borgdorff MW. Treatment outcomes by drug resistance and HIV status among tuberculosis patients in Ho Chi Minh City, Vietnam.Int J Tuberc Lung Dis. 2006 Jan;10(1):45-51.

5. Sarin R, Singla R, Visalakshi $P$, Jaiswal A, Puri MM, Khalid UK, et al. Smear microscopy as surrogate for culture during follow up of pulmonary MDR-TB patients on DOTS Plus treatment. Indian J Tuberc. $2010 \mathrm{Jul} ; 57(3): 134-40$.

6. Gammino VM, Taylor AB, Rich ML, Bayona J, Becerra MC, Bonilla C, et al. Bacteriologic monitoring of multidrugresistant tuberculosis patients in five
DOTS-Plus pilot projects. Int $\mathrm{J}$ Tuberc Lung Dis. 2012 Oct;15(10):1315-22.

7. Bawri S, Ali S, Phukan C, Tayal B, Baruwa P. A study of sputum conversion in new smear positive pulmonary tuberculosis cases at the monthly intervals of $1,2 \& 3$ month under directly observed treatment, short course (dots) regimen. Lung India. 2008 Jul;25(3):118-23.

8. Telenti A, Honore N, Bernasconi C, March $\mathrm{J}$, Ortega A, Heym B, et al. Genotypic assessment of isoniazid and rifampin resistance in Mycobacterium tuberculosis: a blind study at reference laboratory level. J ClinMicrobiol. 1997 Mar;35(3):719-23.

9. Huyen MN, Cobelens FG, Buu TN, Lan $\mathrm{NT}$, Dung NH, Kremer $\mathrm{K}$, et al. Epidemiology of isoniazid resistance mutations and their effect on tuberculosis treatment outcomes. Antimicrob Agents Chemother. 2013 Aug;57(8):3620-7.

10. Palacios E, Franke M, Munoz M, Hurtado $\mathrm{R}$, Dallman $\mathrm{R}$, Chalco $\mathrm{K}$, et al. HIVpositive patients treated for multidrugresistant tuberculosis: clinical outcomes in the HAART era. Int $\mathbf{J}$ Tuberc Lung Dis. 2012;16(3):348-54.

11. Moh. Guidelines For The Management Of Drug Resistant Tuberculosis In Kenya. 2010. 2010.

12. NLTLD. Annual Report.Ministry of Health, National Leprosy, TB and Lung Disease Program (NLTLD). 2014.

13. Sitienei J, Kipruto H, Mansour O, Ndisha M, Hanson C, Wambu R, et al. Correlates of default from anti-tuberculosis treatment: a case study using Kenya's electronic data system. Int J Tuberc Lung Dis. 2015 Sep;19(9):1051-6.

14. Atif M, Sulaiman SA, Shafie AA, Ali I, Asif M, Babar ZU. Treatment outcome of new smear positive pulmonary tuberculosis patients in Penang, Malaysia. BMC Infect Dis. 2014;14:399. 
15. Charles M, Vilbrun SC, Koenig SP, Hashiguchi LM, Mabou MM, Ocheretina $\mathrm{O}$, et al. Treatment outcomes for patients with multidrug-resistant tuberculosis in post-earthquake Port-au-Prince, Haiti. Am J Trop Med Hyg. 2014 Oct;91(4):715-21.

16. Menzies D, Benedetti A, Paydar A, Martin I, Royce S, Pai M, et al. Effect of duration and intermittency of rifampin on tuberculosis treatment outcomes: a systematic review and meta-analysis. PLoS Med. 2009 Sep;6(9):e1000146. 